\title{
OLUSTRUM
}

\section{What makes Expedited Partner Therapy (EPT) and Accelerated Partner Therapy (APT) work for partner notification for bacterial STIs? A systematic review of interventions}

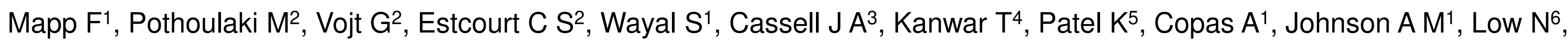
Mercer $\mathrm{C} \mathrm{H}^{1}$, Roberts $T \mathrm{E}^{7}$, Saunders $\mathrm{J}^{1}$, Symonds $\mathrm{M}^{8}$, Flowers $\mathrm{P}^{2}$

${ }^{1}$ University College London, ${ }^{2}$ Glasgow Caledonian University, ${ }^{3}$ Brighton and Sussex Medical School, ${ }^{4}$ University of Cambridge, ${ }^{5}$ Queen Mary, University of London, ${ }^{6}$ University of Bern, ${ }^{7}$ University of Birmingham, ${ }^{8}$ Barts Health NHS Trust

\section{What are EPT and APT interventions?}

Expedited Partner Therapy (EPT) treats the sex partners of persons with STIs by providing prescriptions or medications without prior clinical evaluation. EPT has been shown to reduce STI re-infection and treat a higher proportion of sex partners. EPT which includes remote medical assessment of sexual partners is known as Accelerated Partner Therapy (APT) and meets UK prescribing guidance.

\section{Aim}

To understand the sequential active behaviour change components of EPT/APT and their use of theory in order to optimise and translate interventions for UK services within a forthcoming randomised controlled trial $(\mathrm{RCT})$.

\section{Results}

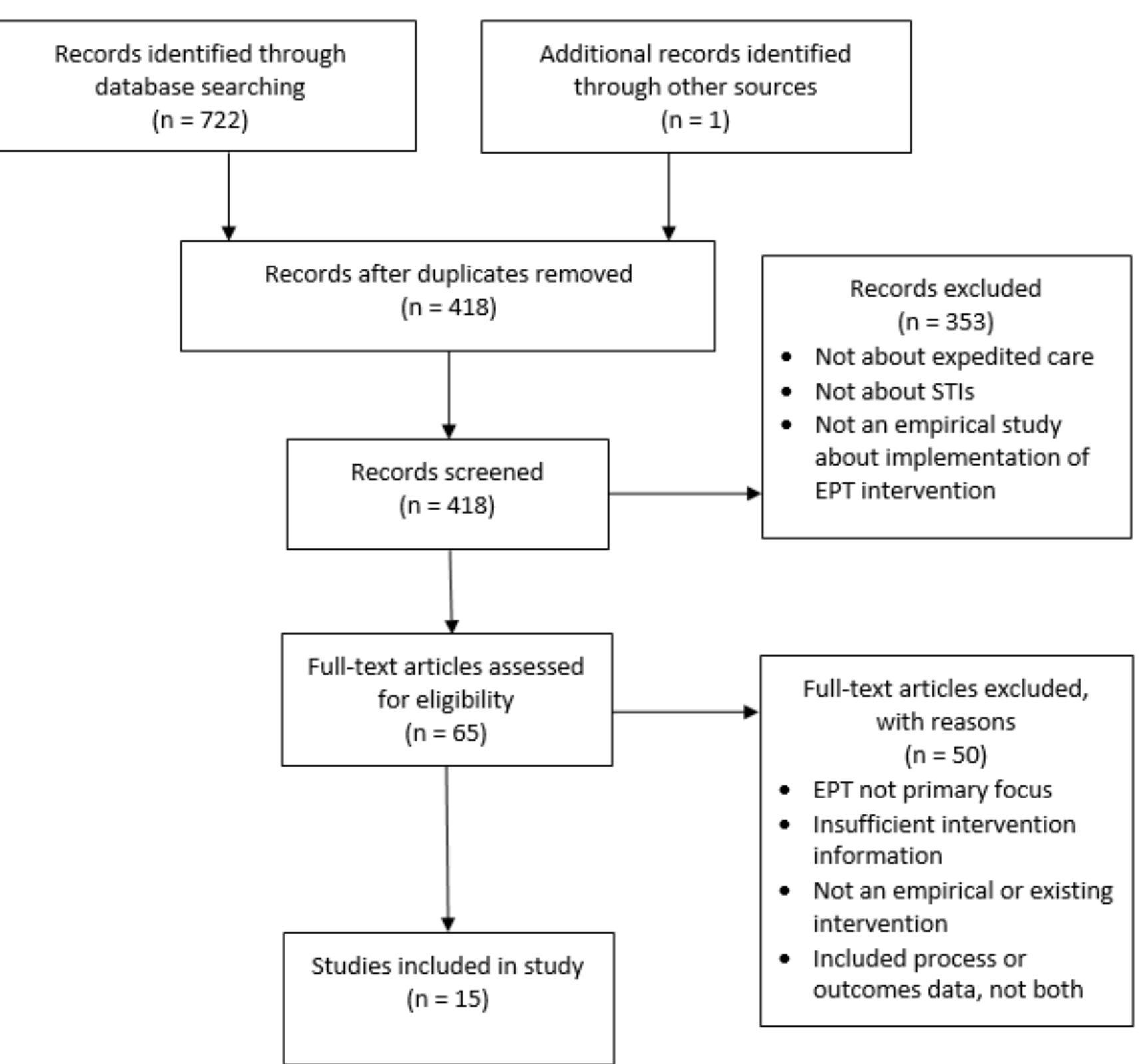

This poster presents independent research funded by the National Institute for Health Research (NIHR) under its Programme Grants for Applied Research Programme (Reference Number RP-PG-0614-20009). The views expressed are those of the author(s) and not necessarily those of the NHS, the NIHR or the Department of Health.

\section{Methods}

We searched Ovid SP MEDLINE, Ovid SP EMBASE, CINAHL Plus, Cochrane Library, NHS Evidence, Open Grey, Scopus and Web of Science in July 2016. Data were extracted about the population, context, intervention components and behaviour change techniques (BCTs).

\begin{tabular}{|l|l|}
\hline Study designs & $\begin{array}{l}\text { 7 RCTs, 2 evaluations, 2 retrospective } \\
\text { observational, 1 non-randomized comparison, 2 } \\
\text { qualitative, 1 pilot }\end{array}$ \\
\hline Study date/setting & $1996-2013$; UK and USA \\
\hline Populations & Women and men (excluding MSM) aged 14+ \\
\hline STIs targeted & Mainly CT, GC (+NGU and TV) \\
\hline Primary outcomes & $\begin{array}{l}\text { Intervention uptake, \% partners treated, re- } \\
\text { infection rates, acceptability }\end{array}$ \\
\hline BCTs & $\begin{array}{l}\text { Interventions involve complex sequences of } \\
\text { behaviours targeting patients, sex partners and } \\
\text { healthcare professionals; consistent range of } \\
\text { BCTs used including 'how to perform a behavior' } \\
\text { and 'information about health consequences' }\end{array}$ \\
\hline
\end{tabular}

\section{Conclusions}

- EPT/APT interventions are atheoretical, developed in response to patient/provider needs.

- Developing an explicit theoretical framework using identified BCTs to guide our intervention development will help in training healthcare professionals to deliver EPT/APT, improving generalisability of interventions and partner notification outcomes. 